\title{
Research of dynamics of turning of machine-tractor aggregate with tractor on wheeled-crawler mover
}

\author{
Marat Fashutdinov ${ }^{1}$, Kamil Khafizov ${ }^{1}$, Ilgiz Galiev ${ }^{1,{ }^{*}, \text { Fanil Gabdrafikov }^{2} \text {, and Farit Khaliullin }}{ }^{1}$ \\ ${ }^{1}$ Kazan State Agrarian University, 420015 Kazan, Russia \\ ${ }^{2}$ Bashkirian State Agrarian University, 450001 Ufa, Russia
}

\begin{abstract}
In the article theoretical preconditions of a description of dynamics of manoeuvrability of machine-tractor, aggregates with a wheeled-tracked mover are considered. For a machine-tractor aggregate with half-tracked progress theoretical formulas of determination of an actual turning radius, the moment of resistance of turn and torque for rotation are obtained. The theoretical preconditions are confirmed by experimental research of the manoeuvrability of the machine-tractor aggregate with the tractor on a halftracked progress, made as the experimental sample. The dependences of the turn coefficient and the resistance coefficient of the turn are obtained, and the correlation coefficients and their significance have confirmed the existence of a stable connection between the changing parameter and the response function. Proceeding from theoretical and experimental research, it is possible to draw a conclusion that the manoeuvrability of the tractor with a wheeled-crawler mover does not concede to the tractor in the basic execution.
\end{abstract}

\section{Introduction}

The main task of agricultural production of the country is to provide the population with food and raw materials of various industries, the implementation of which is directly related to the transfer of agro-industries to an industrial basis. This, in turn, requires a radical improvement in their material and technical equipment, both quantitatively and qualitatively. However, in recent years, enterprises producing tractors and various agricultural machinery have been in dire economic straits. On the other hand, as the statistics shows, the volume of agricultural production is increasing. In subsequent years, this process continued, and as a result, the need of agriculture for tractors and harvesters will $5 . . .7$ times exceed the level of their production [1-4].

In agriculture, the production process is intermittent, which creates certain difficulties for the uniformity of loading of specialized equipment and performance of technological operations in the agro-technical time. The solution of this problem is a flexible changeover of the tractor, its versatility, that is, its fast re-tooling for other agricultural works, which are not typical of the basic variant of the tractor.

As practice shows, the less there are the tractors of different types and grades in one economy, the less there is necessary material and technical base on maintenance of their efficiency that influences, in the end, the cost of agricultural products [5]. In this regard, agriculture needs versatile tractors, which could easily and quickly be rebuilt constructively to perform works not typical of the basic version. In our opinion, the solution of the problem is to create a tractor on a semi-track based on MTZ.

\section{Conditions, materials and methods of research}

When studying the manoeuvrability of the machinetractor aggregate (MTA) with the tractor on the halftracked move, the important point is the determination of the minimum turning radius, the turning moment and the moment of resistance to the turn of the MTA.

The actual turning radius of the MTA depends on the ratio of the turning moment and the moment of resistance to the MTA turning. The greater the turning moment and the less the torque of the MTA, the smaller the actual turn radius of the MTA, which tends to the value of the geometric radius.

To determine the turning moment and the moment of resistance to the turn, the MTA conducted theoretical studies by schematic simulation of the turn of the tractor with a wheel-track specifying the active forces on the tractor.

For confirmation of theoretical preconditions it is necessary to conduct experimental research. Since the maneuverability of the MTA significantly depends on the physical and mechanical characteristics of the soil, the following indicators of soil were determined for the experiment: density, humidity and hardness. The turning radius, the torque, the moment of resistance to the turn of the MTA were determined both on the basis of the serial and on the basis of the experimental tractor of MTZ80PG.

Corresponding author: drgali@mail.ru 
To study the dynamics of turning was developed experimental installation with tractor with a wheeledcrawler mover on the basis of tractor MTZ-80.

Experimental studies were conducted in the conditions of the land of Kazan state agrarian university. The condition of the soil during the experiments was controlled by density, humidity and hardness. Plots for experiments were selected horizontally, with a relative smooth surface. An agrotechnical structure of field surface includes a stubble, raw field, freshly ploughed field.

In the course of studies the values of the actual turning radii of the MTA were measured under different conditions (agrotechnical structure of the field surface, the angle of rotation of the steered wheels).

\section{The results of the study}

The geometric turning radius of the MTA (Fig. 1) depends on the angle of rotation of the steered wheels [6]:

$$
R_{r}=L \cdot \operatorname{ctg} \alpha
$$

$R_{r}$ - geometric turning radius of the MTA, m; $L-$ distance between axles of leading and driven tractor wheels, $\mathrm{m} ; \alpha-$ the average angle of rotation of the steered wheels, degree.

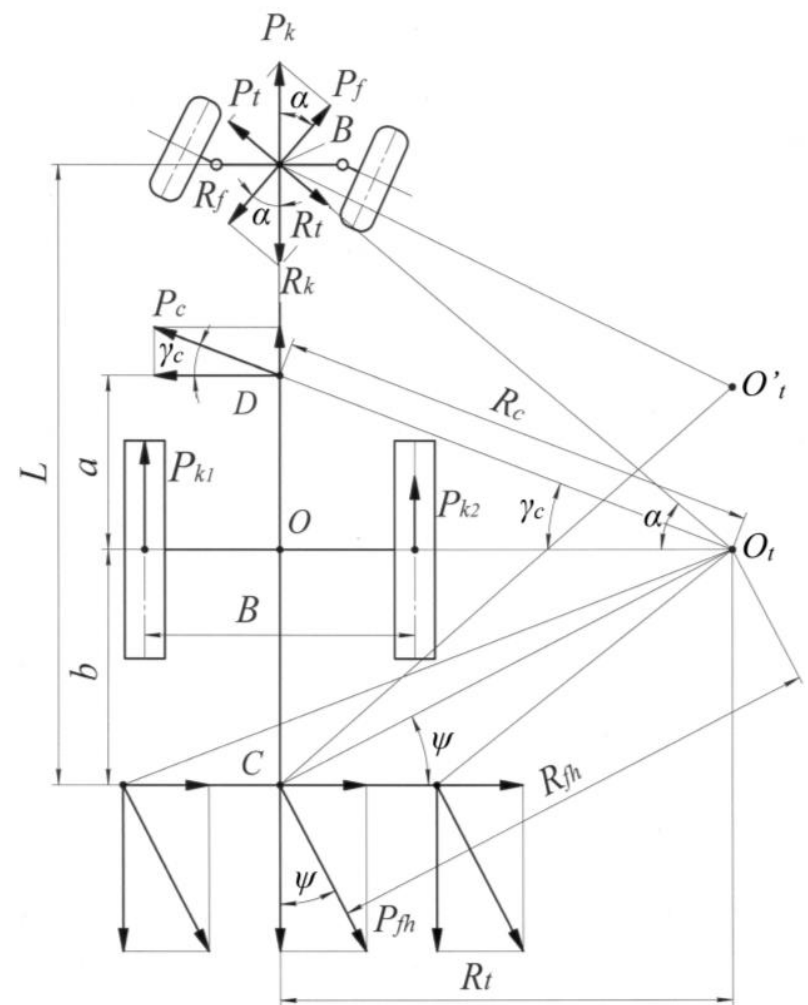

Fig. 1. The scheme of forces acting on the tractor with the wheeled-crawler mover at its turning in the composition of the MTA.

The actual radius of the turn of the MTA with the wheeled-crawler mover, unlike the geometric radius of rotation, depends on many factors: the coefficient ofcoupling of the mover with the soil, the coefficient of rolling resistance, the speed of the movement of the MTA, the distance between the tractor tracks, the force on the hook, the longitudinal distance between the axles of the tractor, the average angle of rotation of the steered wheels, the redistribution of weight between the front and rear axles of the tractor, the redistribution of weight between the outer and inner wheels, the length of the supporting surface caterpillar with soil, humidity and soil density, the stress of soil compaction, the design parameters of the front steered wheels.

The MTA moves at a constant speed and on a circular trajectory with a constant turning radius. The tangential traction force of the rear caterpillars is transferred to the tractor's skeleton in the form of the equivalent force of the $P_{K}$ (Fig. 1), which is directed forward along the axis of the tractor. This pushing force of $P_{K}$ is transferred to the front axle and front wheels.

In the spot of the contact of the controlled wheel with the soil, there are reactions, the equivalent force of which equals the pushing force of the $P_{\kappa}$ and opposite it is directed. Each of these forces can be decomposed into two components. The reactive force component $R_{f}$ of the $R_{\kappa}$ is the force of wheel rolling resistance. The force component $R_{t}$ the force creates a turning moment of the MTA $M_{t}$ around the point "O":

$$
M_{t}=R_{t} \cdot \cos \alpha \cdot L=G_{t} \cdot \phi_{c} \cdot \cos \alpha \cdot L,
$$

$G_{t}-$ vertical load on the front axle of the tractor, $\mathrm{N} ; \varphi_{\mathrm{c}}-$ wheel coupling coefficient with soil.

In addition, the following forces also operate on the tractor: centrifugal force of the $P_{c}$, which arises as a result of moving the tractor carrier system with some angular velocity $\omega_{t}$ around the centre of the rotation point "O $\mathrm{O}_{\mathrm{t}}$ "; tangent forces of the $P_{K l}$ and $P_{K 2}$, respectively on the running and lagging tracks; force on the hook of the $P_{f h}$.

The moment of resistance to the turning of $M_{r t}$ around the point "O":

$$
\begin{aligned}
& M_{r t}=R_{f} \cdot \sin \alpha \cdot L+P_{c} \cdot \cos \gamma_{c} \cdot a+ \\
& +\frac{\mu \cdot G_{l} \cdot L_{c a t}}{4}+P_{f h} \cdot \sin \psi \cdot b
\end{aligned}
$$

$\gamma_{c}$ - the angle between the direction of centrifugal force of the $P_{c}$ and the line passing through the center of rotation "O $\mathrm{O}_{\mathrm{t}}$ " and point "O", deg.; $a$ - distance from the rear axle to the center of gravity of the tractor, $\mathrm{m} ; \mu$ - the cast to value of a coefficient of resistance to turn; $G_{l}$ vertical load on the rear axle of the tractor, $\mathrm{kN} ; \psi$ - angle between the direction of action of the hook force of the $P_{f h}$ and the axial line of the tractor, deg.; $b$ - distance from the rear axle to the coupling device (hook), $\mathrm{m}$.

The strength of wheel rolling resistance depends on soil properties and vertical load on steered wheels:

$$
R_{f}=G_{t} f_{r}
$$

$f_{r}$ - coefficient of wheel rolling resistance on the soil.

The centrifugal force of the $P_{c}$ is known to be directly proportional to the weight of the tractor, the 
square of the rate of movement of the MTA and inversely proportional to the radius of the center of gravity rotation:

$$
P_{c}=\frac{G v^{2}}{g \cdot R_{c}},
$$

$G$ - tractor weight, $\mathrm{kN} ; g$ - acceleration of the free fall,

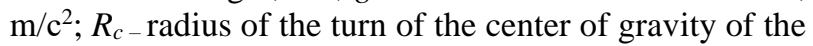
tractor, $\mathrm{m}$.

The radius of rotation of the tractor center of gravity is always slightly larger than the geometric turning radius $R_{r}$ and with the formula (1):

$$
R_{c g}=\sqrt{a^{2}+R_{r}^{2}}=\sqrt{a^{2}+L^{2} \cdot \operatorname{ctg}^{2} \alpha} .
$$

Therefore, equation 8 will take the following form:

$$
P_{c}=\frac{G v^{2}}{g \cdot \sqrt{a^{2}+L^{2} \cdot \operatorname{ctg}^{2} \alpha}} .
$$

Let us consider the triangle with the vertices of the $\mathrm{DSO}_{\mathrm{t}}$, from which we define the formula component (3):

$$
\cos \gamma_{c}=\frac{R_{r}}{R_{c}}=\frac{L \cdot \operatorname{ctg} \alpha}{\sqrt{a^{2}+L^{2} \cdot \operatorname{ctg}^{2} \alpha}} .
$$

The cast to value of a coefficient of resistance to turn $\mu$ depends on physical and mechanical properties of a soil, structure of caterpillars and depth of their immersion in soil. However, the maximum influence on this coefficient is the turning radius (the smaller the turning radius, the greater the coefficient of $\mu$ ).

In the indicative calculations of the value of coefficient $\mu$ at different radii of turning, it is possible to apply the empirical formula of A. O. Nikitin [7]:

$$
\mu=\frac{\mu_{\max }}{\left[c+(1-c) \cdot\left(\rho_{t}+0,5\right)\right]},
$$

$\mu_{\max }-$ the highest value of the coefficient of resistance turn, (the denser the soil, the smaller the value of the coefficient $\left.\mu_{\max }(0.7 \ldots 1.0)\right) ; \rho_{t}$ - relative turning radius; $c$ - depends on soil conditions (the denser the soil, the greater the value of $c(0.75 \ldots 0.9))$.

The relative radius of rotation is determined by the formula:

$$
\rho_{t}=\frac{R_{r}}{B} .
$$

$B$ - distance between the tractor's gusinians, $\mathrm{m}$.

In movement of the MTA along the curvilinear trajectory the on-hook force of the $P_{f h}$ will be directed not to an axis of the tractor, and under some angle $\psi$. Breakdown of the on-hook force of the $P_{f h}$ into two components: transverse $P_{f h} \cdot \sin \psi$ and longitudinal $P_{f h} \cdot \cos \psi$. The transverse component of the on-hook force is directed towards the lagging caterpillar and in relation to the point of "O" gives the moment of resistance to the turn of the MTA.

Under the action of the longitudinal component of the on hook force the load is redistributed along the axes of the tractor. The redistribution of load on axes, first of all, depends on the type of agricultural machine and the way of regulation.

The vertical load on the front axle, taking into account the effort on the hook, will be:

$$
G_{t}=G_{t}^{0}-P_{f h} \frac{h_{h}}{L},
$$

$G_{t}^{0}$ - vertical load on the front axle without taking into account the on hook force, $\mathrm{kN} ; h_{h}$ - distance from the ground to the point of the trailer, $\mathrm{m}$.

The hook force of the $P_{f h}$ will load the rear axle as follows:

$$
G_{l}=G_{l}^{0}+P_{f h} \frac{h_{h}}{L}+P_{f h} \cdot \operatorname{tg} \beta,
$$

$G_{l}^{0}$ - the vertical load on the rear axle without regard to the on hook force, $\mathrm{kN} ; \beta$ - angle between the horizontal plane and the line of traction force, deg.

Thus, the hook force of the $P_{f h}$, loading the rearleading caterpillar, increases traction properties of the tractor, but at the same time, unloading the front driven wheels, worsens the controllability of the MTA.

The angle between the direction of the action of the $P_{f h}$ and the axial line of the tractor $\psi$ depends on the angle of rotation of the steered wheels $\alpha$, namely - the greater the angle of $\alpha$, the greater the angle of $\psi$.

The turning radius of the trailer point of the $R_{f h}$ is slightly larger than the geometric radius of the $R_{r}$ (see figure 1):

$$
R_{f h}=\sqrt{b^{2}+R_{r}^{2}}=\sqrt{b^{2}+L^{2} \cdot \operatorname{ctg}^{2} \alpha},
$$

Let us consider the triangle with the vertices of the $\mathrm{SO}_{\mathrm{t}} \mathrm{E}$, from where we define the component of the formula (3), while taking into account that the shift of the center of turning (from point "O" to point " $O_{t}$ ") will change the angle of $\psi$, and therefore change the value of $\sin \psi$ and this change can be taken into account by the introduction in the formula of correction coefficient $\mathrm{k}$ :

$$
\sin \psi=\frac{k \cdot b}{\sqrt{b^{2}+L^{2} \cdot \operatorname{ctg}^{2} \alpha}} .
$$

It is difficult to determine exactly the $\mathrm{k}$ coefficient value. Therefore, we assume that the center of turning is shifted in this way (see Figure 1):

$$
\frac{C O_{t}^{\prime}}{B O_{t}^{\prime}}=\frac{L}{b}=\mathrm{k}
$$

Subject to expressions $4 \ldots 14$, formula 3 will take the following form:

$$
\begin{aligned}
& M_{r t}=\left(G_{t}^{0}-P_{f h} \frac{h_{h}}{L}\right) \cdot \sin \alpha \cdot L+\frac{G v^{2} \cdot L \cdot \operatorname{ctg} \alpha \cdot a}{g \cdot a^{2}+L^{2} \cdot \operatorname{ctg}^{2} \alpha}+ \\
& +\frac{\mu_{\text {мax }} \cdot L_{c a t} \cdot\left(G_{l}^{0}+P_{f h} \frac{h_{h}}{L}+P_{f h} \cdot \operatorname{tg} \beta\right)}{4 \cdot\left[c+(1-c) \cdot\left(\frac{R_{r}}{B}+0,5\right)\right]}+\frac{P_{f h} \cdot L \cdot b}{\sqrt{b^{2}+L^{2} \cdot \operatorname{ctg}^{2} \alpha}} .
\end{aligned}
$$

Let us introduce a concept such as the coefficient of turning of the $K_{R}$, which is equal to the ratio of the actual radius to the geometric:

$$
K_{R}=\frac{R_{f}}{R_{r}},
$$

$R_{f}$ - the actual turning radius of the MTA, m.

When $K_{R}=1$, the ability to rotate is considered normal, when the $K_{R}<1-$ redundant and at the $K_{R}>1-$ insufficient. 
The ratio of the moment of resistance of the turn and the turning moment shall be denoted as a factor of resistance to rotation $K_{m}$ :

$$
K_{m}=\frac{M_{r t}}{M_{t}} .
$$

Using formulas 1,2 and 16 , the geometric radius values are obtained, turning moments and moments of resistance turn. As a result of joint mathematical processing on the developed program on the computer $[8,9]$, empirical formulas of dependence of the coefficient of turning from the factor of resistance of a turn on various agrotechnical structure of the field surface have been received. Force on a hook changed from 0 to $10 \mathrm{kN}$, at the speed of the MTA movement of $2 \mathrm{~m} / \mathrm{s}$, which are presented in table 1 . The relationship between the changing parameter and the response function was determined by the correlation coefficient. The coefficient value is evaluated by the correlation coefficient error $m_{R}$ indicator from the following condition [10]:

$$
R \geq 3 \cdot m_{R}
$$

Table 1. Empirical dependence of the coefficient of turning and the factor of resistance turn.

\begin{tabular}{|c|c|c|c|}
\hline \multirow{2}{*}{$\begin{array}{c}\text { Agrotechnical } \\
\text { structure of field } \\
\text { surface }\end{array}$} & 14 & \multicolumn{3}{|c|}{ Steering wheel rotation angle, deg. } \\
\cline { 2 - 4 } & & 20 & 27 \\
\hline Raw field & $K_{R}=1,72-3,62 K_{m}+4,15 K_{m}^{2}$ \\
$\left(R=0,65 ; \mathrm{m}_{R}=0,21\right)$ & $\begin{array}{l}K_{R}=1,625+\mathrm{Lg} K_{m} \\
\left(R=0,71 ; \mathrm{m}_{R}=0,24\right)\end{array}$ & $\begin{array}{c}K_{R}=1,15+10,87 K_{m}^{6,51} \\
\left(R=0,48 ; \mathrm{m}_{R}=0,13\right)\end{array}$ \\
\hline Stubble & $K_{R}=\frac{K_{m}}{0,22+0,48 K_{m}}$ & $\begin{array}{l}K_{R}=1,05+94,9 K_{m}^{10,51} \\
\left(R=0,51 ; \mathrm{m}_{R}=0,12\right)\end{array}$ & $\begin{array}{l}K_{R}=4,34-12,42 K_{m}+11,94 K_{m}^{2} \\
\left(R=0,64 ; \mathrm{m}_{R}=0,18\right)\end{array}$ \\
& $\left(R=0,68 ; \mathrm{m}_{R}=0,15\right)$ & $K_{R}=1,235-1,16 K_{m}+2,05 K_{m}^{2}$ & $K_{R}=7,9-18,64 K_{m}+12,74 K_{m}^{2}$ \\
& $K_{R}=3,65-7,48 K_{m}+5,48 K_{m}^{2}$ \\
$\left(R=0,53 ; \mathrm{m}_{R}=0,12\right)$ & $\left(R=0,72 ; \mathrm{m}_{R}=0,2\right)$ & \\
\hline
\end{tabular}

The study of the maneuverability of both types of MTA came down to the fact that the actual radii of turns were measured at various operational factors.

\section{Conclusion}

The necessity of universal tractors is grounded, which can be easily and quickly reconstructed for the performance of works not typical for the basic version of this tractor.

Experimental research on different agrotechnical structures of the field surface, hook loads and rotation angles of control wheels was carried out on the pilot sample of the tractor with wheeled-tracked progress.

The dependencies of the turning coefficient and the resistance factor have been obtained, and the correlation coefficients and their significance have confirmed the existence of a stable connection between the changing parameter and the response function.

Eith equal operational conditions the actual turning radius of the MTA with the tractor with the wheel-track stroke, as a whole, exceeds the analogous indicator of the MTA with the tractor with the wheel stroke by $3.5 \%$.

In the raw field and freshly ploughed field at the hook loads of more than $5 \ldots 6 \mathrm{kN}$, the turn radius of the MTA with the tractor on the wheel-track move begins to increase more intensively than that at the MTA with the basic tractor. The inverse picture is observed on the stubble - the actual turning radius of the MTA with the base tractor, under hook loads over $5 \mathrm{kN}$, starts to grow more intensively and at some operational factors exceeds the actual turning radius of the MTA with the tractor on a wheel-track move.

\section{References}

1. R.N. Khafizov, K.A. Khafizov, A.A. Nurmiev, I.G. Galiev, 17th ISC Engineer. for rural developm. Proc., 17, 168 (2018)

2. I.G. Skobtsov, A.V. Pitukhin, M.I. Kulikov, Resources and Technology (2008)

3. S.S. Yuferev, Technical sciences in Russia and abroad, mater. 3th ISC, 38, 136 (2014)

4. R.A. Valiev, R.I. Safin, N.I. Semushkin, B.G. Ziganshin, Herald of Kazan state agricult. Univer., 7(4), 65 (2012)

5. V. Guskov, N. Velev, Yu. Atamanov, Tractors (1988)

6. G.M. Kutykov, Theory of tractor and automobile (1996)

7. V.A. Skurnikov, A.A. Maschensky, A.S. Solinsky, Fundamentals of theory and calculation of the tractor and the car (1986)

8. A.P. Karabanitsky, O.A. Levuukova, Theoretical substantiation of parameters of energy-saving machine-tractor aggregate (2014)

9. I.G. Galiev, K.A. Khafizov, N.R. Adigamov, R.K. Khusainov, 17th ISC Engineer. for rural developm. Proc., 17, 373 (2018)

10. O.P. Krastin, Methods of regression and correlation analysis (1970) 